Fish and Fisheries

January 2019, Volume 20, Issue 1, Pages 189-198

https://doi.org/10.1111/faf.12338

Archimer

https://archimer.ifremer.fr/doc/00470/58162/

\title{
Estimating effective population size of large marine populations, is it feasible?
}

\author{
Marandel Florianne ${ }^{1,{ }^{*}}$, Lorance Pascal ${ }^{1}$, Berthelé Olivier ${ }^{1}$, Trenkel Verena ${ }^{1}$, Waples Robin S. ${ }^{2}$, \\ Lamy Jean-Baptiste ${ }^{3}$
}

${ }^{1}$ Ifremer; Ecologie et Modèles pour l'Halieutique; Nantes ,France

${ }^{2}$ Northwest Fisheries Science Center; National Marine Fisheries Service; NOAA; Seattle Washington, USA

${ }^{3}$ Ifremer; Génétique et Pathologie des Mollusques Marin; La Tremblade ,France

*Corresponding author : Florianne Marandel, email address : florianne.marandel@ifremer.fr

\begin{abstract}
:
stainable exploitation of marine populations is a challenging task relying on information about their current and past abundance. Fisheries-related data can be scarce and unreliable making them unsuitable for quantitative modelling. One fishery independent method that has attracted attention in this context consists in estimating the effective population size $(\mathrm{Ne})$, a concept founded in population genetics. We reviewed recent empirical studies on $\mathrm{Ne}$ and carried out a simulation study to evaluate the feasibility of estimating $\mathrm{Ne}$ in large fish populations with the currently available methods. The detailed review of 26 studies found that published empirical $\mathrm{Ne}$ values were very similar despite differences in species and total population sizes $(\mathrm{N})$. Genetic simulations for an age-structured fish population were carried out for a range of population and samples sizes, and Ne was estimated using the Linkage Disequilibrium method. The results showed that already for medium-sized populations (1 million individuals) and common sample sizes (50 individuals), negative estimates were likely to occur which for real applications is commonly interpreted as indicating very large (infinite) Ne. Moreover, on average, Ne estimates were negatively biased. The simulations further indicated that around $1 \%$ of the total number of individuals might have to be sampled to ensure sufficiently precise estimates of Ne. For large marine populations, this implies rather large samples (several thousands to millions of individuals). If however such large samples were to be collected, many more population parameters than only $\mathrm{Ne}$ could be estimated.
\end{abstract}

Keywords : census population size, effective population size, fish, linkage disequilibrium, management, simulation 


\section{$1 \quad N_{E}$ ESTIMATION FOR LARGE MARINE POPULATIONS}

Fishery science is driven by the need to produce scientific advice for the management and conservation of marine resources and ecosystems (Dankel and Edwards 2016). This motivates the collection of information on population status and biology. Increasingly, attention is paid to the genetic state of marine populations (Ovenden et al. 2015) with numerous studies being published on genetic diversity (Bryan-Brown et al. 2017), genetic population connectivity (Bryan-Brown et al. 2017), and genetic population size (Luikart et al. 2010). For example, data from the Web of Science (WoS) show that between 2000 and 2017, the annual number of publications estimating effective population size $\left(N_{e}\right)$ of marine species increased six fold (Fig. S1 in supporting information, Web of Sciences). Theoretically from a genetic point of view, $N_{e}$ is defined as the size of an ideal population that is experiencing the same rate of change in allele frequencies or heterozygosity as the observed population (Luikart et al. 2010). Ideal populations are made of diploid organisms with sexual reproduction, non overlapping generations, random mating, no migration, no mutation, but also no natural selection (Wright 1931). Effective population size is considered a pertinent parameter for management as it relates to rates of genetic drift and loss in genetic variation (Hare et al. 2011). Moreover, $N_{e}$ is a useful concept for evaluating the genetic future of marine populations (harvested or not) as reductions in $N_{e}$ are positively correlated with reductions in population viability (Soule 1987).

The use of $N_{e}$ in scientific studies has increased (Wang 2005, Leberg 2005, Luikart et al. 2010, Supplementary figure 1) which can be linked to the increased availability of molecular markers but also the continual improvement of estimation methods (Luikart et al. 2010; Wang 2016; Waples et al. 2016). In the past, $N_{e}$ was considered difficult to estimate but this situation has changed (Schwartz et al. 1998; Leberg 2005). As a consequence, $N_{e}$ is nowadays commonly estimated for varied marine taxa: mammals (DeWoody et al. 2017), crustaceans (Watson et al. 2016), corals (Holland et al. 2017) and fishes (Laconcha et al. 2015; Zhivotovsky et al. 2016; Pita et al. 2017). Among commercial fish species, both target (Poulsen et al. 2005; Montes et al. 2016) and bycatch species (Chevolot et al. 2008) have been studied, representing a wide range of life history strategies, habitats, population 
structures but also census population sizes (i.e. total number of individuals in the population including immatures, denoted $N$ ), from hundreds to billions of individuals.

Many marine fish populations are very large compared to vertebrates but also present a large variety of reproductive strategies. In ideal populations as defined above all individuals have the same reproductive success making $N_{e}$ equal to $N$. Natural populations do not have all properties of ideal populations, leading to variance in the reproductive success of individuals implying that some individuals can contribute genetically more to the next generation than others. Thus, in most natural populations $N_{e}$ is smaller than $N$.

Genetic simulations for ideal populations indicated that $N_{\mathrm{e}}$ might not be reliably estimated for medium sized populations $\left(N_{\mathrm{e}}>10^{6}\right)$, independent of sample size (Waples 2016). In this seminal study Waples (2016) investigated two hypotheses which could lead to too small $N_{\mathrm{e}}$ estimates for large populations: unequal reproductive success (sweepstakes hypothesis, Hedgecock 1994) or biased estimation. He concluded that for the biological explanation to hold, few individuals would need to be responsible for most of the successful reproduction, i.e. the variance in reproductive success of sameage, same-sex individuals has to be orders of magnitude higher than the mean. Without ruling-out the sweepstake hypothesis, Waples (2016) suggests that biased estimation seemed to be a likely cause for creating small $N_{\mathrm{e}}$ estimates for large populations.

To evaluate the success in estimating $N_{\mathrm{e}}$ for natural marine populations, we analyzed 26 studies containing 55 empirical estimates of $N_{\mathrm{e}}$ for fish or crustaceans (tables S1 and S2 in supporting information). These studies correspond to all relevant studies published in 2016 or 2017 and the most cited studies for 2000 to 2015. Studies were separated into two categories according to the main goal of the study: estimating $N_{e}$ (20 estimates in 14 studies) or other genetic questions ( 34 estimates in 12 studies). For studies estimating $N_{e}$ as a side goal, sample sizes were smaller compared to studies estimating $N_{e}$ as the main goal (313 mean \& $19-1833$ 95\% range for side goal; 3481 and 50-4063 95\% range for main goal; Fig 1a \& c). Few studies in either category used sample sizes larger than 500 individuals (25\% side goal; $43 \%$ main goal; Fig 1 . a \& c). Only studies estimating $N_{e}$ as a side goal reported negative or infinite $N_{e}$ estimates (Fig 1.c \& d). These negative or infinite $N_{e}$ estimates corresponded to low sample sizes ( $<50$ individuals) or very large $N$ ( $>1$ billion individuals). In the 
reviewed studies $N$ ranged from thousands of individuals (Zebra shark, southern Queensland Australia, Dudgeon and Ovenden 2015) to several billions (European anchovy in the Bay of Biscay, Montes et al. 2016) (Fig. 1b). No significant linear relationship was found between $N_{e}$ and either $N$ or sample size $S$. This was tested using a linear model with only main effects and data from the 11 studies for which $N$ was available. The absence of relationship between $N_{e}$ estimates and $N$ seems to corroborate the simulation results obtained by Waples (2016), in particular the conclusion that $N_{\mathrm{e}}$ estimates for large populations can be biased to the point of becoming meaningless.

Several factors impact $N_{\mathrm{e}}$ estimates, while increasing sample size generally improves their accuracy and precision (Waples and Do 2010). However, for marine populations, obtaining a large number of samples (tissue, scales...) can be difficult and genotyping costs can also limit sample sizes. As a consequence most sample sizes were under 1000 individuals in the reviewed studies (Fig. 1a \& c). This led to sample sizes corresponding to less than $1 \%$ of the census population (for example, $8 \mathrm{E}-$ 06\%, for North Sea cod Poulsen et al. 2005; 2E-07\% for plaice Hoarau et al. 2005, 2E-04\% for European sardine Laurent and Planes 2007). Macbeth et al. (2013) showed by simulation that for the narrow-barred Spanish mackerel a sample size of 5000 individuals was necessary to estimate $N_{e}$ of a population with census size $N=10000$ using the Linkage Disequilibrium method (see below for details regarding this method). This result emphasizes the need for appropriate sampling designs for estimating $N_{e}$. Currently there are few recommendations available for appropriate sampling designs for estimating $N_{e}$ as this is expected to be species dependent. For elasmobranchs, Dudgeon et al. 2012 advised that 50 individuals were sufficient for $N_{e}<200$ individuals while in this paper we show by simulation that, for a thornback ray (Raja clavata) like elasmobranch species assuming $N_{e}<100$ ( $N=1000$ individuals), 300 sampled individuals would be needed for precise (though biased) estimation (see below). Other than the sampling design, the type (microsatellites or SNPs) and the number of markers can have a large effect on $N_{e}$ estimates (Waples and Do 2010, F. Marandel unpublished results).

Numerous methods and estimators are available for estimating contemporary $N_{e}$. However, two approaches dominate the field: temporal estimation which requires temporally spaced samples from a population and single-point estimation which requires a sample from only a single point in time. 
Among the two approaches, the most popular method is the single-point Linkage Disequilibrium Method (LDM, Hill 1981, Waples et al. 2014). It was used for 22 estimates among the 55 estimates provided in the reviewed studies, while the Temporal Method (TM, Jorde and Ryman 1995) based on temporal changes in allele frequencies was used for 12 estimates and the Pseudo Likelihood Method (PLM, Wang 2001) also based on temporal changes in allele frequencies for seven estimates. Only 14 estimates used other methods. LDM, TM and PLM have been widely reviewed for various species (Schwartz et al. 1998; Wang 2005; Waples et al. 2014) with emphasis on the need for considering the life history of the studied species to obtain reliable $N_{e}$ estimates or even to be able to interpret correctly $N_{e}$ estimates. An example is the bias induced in $N_{e}$ estimates by overlapping generations (which occurs in a natural population in contrast to an ideal population), i.e. where more than one breeding generation is present at any one time. There are several ways to minimize this bias in TM, notably using a long time lag between temporal samples (for example a generation length) or using a bias correction. Indeed, two decades ago, a correction factor for estimating $N_{e}$ for species with overlapping generations was developed by Jorde and Ryman (1995) for TM. The calculation of this correction factor requires knowledge of life history traits, which might explain why it is not always used.

To further explore the (non-)feasibility of estimating effective population size for large populations using commonly used sample sizes, we present results from a simulation study in the next section. In contrast to Waples (2016) we simulated overlapping generations based on life history traits of thornback ray (Raja clavata), an elasmobranch widely distributed in European waters. Elasmobranchs are generally more vulnerable to fishing than teleosts and have smaller population size. Census population size of this species in the Northeast Atlantic might be millions of individuals (Marandel et al. 2016). Thus elasmobranchs are of interest for $N_{e}$ estimation both in terms of conservation and technical applicability of the method. For $N_{\mathrm{e}}$ estimation we chose the Linkage Disequilibrium method as it is still the most widely-used method. 


\section{GENETIC SIMULATION OF A LARGE POPULATION}

\subsection{Method}

Genetic simulations were set up mimicking thornback ray life history traits, i.e. a low fecundity with medium to high survival (Supplementary table S3). Populations of $N$ individuals were simulated for 151 years but only the last year was used for estimating $N_{e}$. Life history traits were used in two ways as in Waples et al. (2014): (1) to calculate the expected (demographic) effective population size $E\left[N_{e}\right]$ (AgeNe software,Waples et al. 2011), (2) to carry out simulations to obtain age-structured genetic data (simuPOP module, Peng and Kimmel 2005) to which the LDM estimator of $N_{\mathrm{e}}$ was applied (Fig. S2).

The expected (demographic) effective population size per generation $E\left[N_{e}\right]$ was calculated using the AgeNe software based on life history traits (Felsenstein-Hill method, Waples et al. 2011). The method assumes a stable population (thus stable age structure) and constant survival and fecundities at age (Waples et al 2014, eq. 1):

$$
E\left[N_{e}\right]=\frac{4 N_{1} G}{V_{k .}+2}
$$

where $N_{1}$ is the number of age 1 individuals in the population and $G$ is the generation length (= mean age of parents of newborns). Both depend on survival and fecundity rates, in addition $N_{1}$ depends on population size $N . V_{k}$. is the inter-individual variance of lifetime reproductive success; the mean life time reproductive success for a stable population is 2 , hence the 2 in the denominator of equation 1 .

All modeled populations in simuPOP were simulated with a 1:1 sex ratio and random assignment of age at initialization (year 0). Newborn individuals were generated by drawing one male and one female from the pool of potential parents. All potential parents of the same sex and age had an equal probability to become a parent. Two hundred biallelic genetic markers corresponding to SNPs (Single Nucleotide Markers) were simulated with an initial allele frequency of 0.5. Preliminary simulations were conducted with 1600 biallelic genetic markers showing that a plateau in terms of precision and accuracy of $N_{e}$ estimates was reached at around 200 markers.

Four populations sizes were simulated, $N \in(1000,10000,100000,1000000)$ individuals, to evaluate the performance of the LDM for different census sizes. Note that the largest simulated population size was smaller than many real fish populations due to computational constraints. As 
simulated populations contained immatures and overlapping generations with mature individuals reproducing several times, $\mathrm{E}\left[N_{e}\right]$ of each population was smaller than the simulated population size $N$ $\left(\mathrm{E}\left[N_{e}\right]=0.087 N\right.$, Table 1). For each population size, 30 replicates were carried out to capture the stochasticity inherent in genetic simulations. For each population replicate nine sample sizes $S \in(50$, $100,200,300,500,1000,1500,5000,10000$ ), were investigated (Table 1); the larger sample sizes could only be explored for the largest population sizes. Sampled individuals were randomly drawn from newborns in the last year. For each population replicate and sample size, sampling was repeated 50 times, i.e. for each population and sample size there were 1500 simulated data sets. All 200 simulated loci were generally used for estimation, unless the minor allele frequency was $<0.05$ in which case it was removed as suggest by Waples and Do (2010) to minimize sampling bias.

The Linkage Disequilibrium (LD) is the non-random association of alleles at different gene loci, e.g. allele $A$ at SNP locus 1 with allele $b$ at SNP locus 2 . When loci are inherited independently, the frequency of the $A b$ loci association is just the product of the two allele frequencies $P_{\mathrm{A}}$ and $P_{\mathrm{b}}$ in the population. In natural populations, overlapping generations, gene flow and linked loci will influence LD in addition to finite population size.

For applying the LDM, the LD is measured by the co-variance $(D)$ and the squared correlation $\left(r^{2}\right)$ between loci. The squared correlation $r^{2}$ is defined as:

$$
r^{2}=\frac{D^{2}}{P_{A} P_{a} P_{B} P_{b}}
$$

where $A$ and $a$ are the major et minor alleles (in frequency) at SNP locus 1 and $B$ and $b$ are the major et minor alleles at SNP locus 2, $D=P_{A B}-P_{A} P_{B}$ and $P_{A}, P_{a}, P_{B}$ and $P_{b}$ are the frequencies of alleles $A$, $a, B$ and $b$ respectively. $P_{A B}$ is the haplotype (joint) frequency of the gamete/chromosome carrying the allele $A$ at locus 1 and the allele $B$ at the locus 2 . Thus the calculation of LD is based on allele and haplotype frequencies. However in most fishery studies, haplotype frequencies are not available as the data does not contain information on which one of the pair of chromosomes holds which allele making the exact calculation of $r^{2}$ impossible. To circumvent this obstacle, a proxy is used, called the composite measure of linkage disequilibrium. The full explanation of this proxy is out of scope of this article and it reviewed in Hamilton and Cole (2004). 
For estimating $N_{e}$ based on the proxy estimate $\hat{r}^{2}$ adjusted for sample size $S$ related sampling error according to Weir (1979), the following relationship was used (Waples 2006):

$$
\widehat{N}_{e}=\frac{1 / 3^{+} \sqrt{1 / 9^{-2.76 \hat{r}^{2 \prime}}}}{2 \hat{r}^{2 \prime}} \quad \text { with } \quad \hat{r}^{2 \prime}=\hat{r}^{2}-1 / S-3.19 / S^{2}
$$

Equation (3) shows that if $1 / S$ is larger than $\hat{r}^{2}$ a negative estimate of $\widehat{N}_{e}$ is obtained. Thus negative estimates occur when sampling error is larger than the genetic signal (correlation between loci, eq 1), without invoking any genetic effect. The usual practitioner interpretation made is that negative $N_{\mathrm{e}}$ estimates indicate a very large effective population size, hence negative estimates are replaced by infinity (Laurie-Ahlberg and Weir 1979; Nei and Tajima 1981). In reality, negative estimates can also simply be caused by an insufficient sample size.

The estimator in eq 3 is implemented in NeEstimator V2 (Do et al. 2014) which was used for the simulated data sets. As this software does not account for overlapping generations $N_{\mathrm{e}}$ estimates will be biased to an unknown degree depending on the simulated life history (Waples et al. 2014).

Quantifying accuracy (or bias) and precision of estimates of effective population size is complicated because $\widehat{N}_{e}$ has a skewed distribution and can be arbitrarily large (or even negative as discussed above). Accordingly, we followed Wang (2001, 2009), who focused on bias and precision of the inverse $1 / N_{\mathrm{e}}$, which is proportional to the rate of genetic drift and is the signal for effective size that is detected by all genetic estimation methods. The estimates of $1 / N_{\mathrm{e}}$ were then compared to the inverse of the expected value $E\left[N_{e}\right]$ (eq 1). Thus we analyzed the distribution of $\frac{1 / \widehat{N}_{e}}{1 / E\left[N_{e}\right]}=\frac{E\left[N_{e}\right]}{\widehat{N}_{e}}$. Note that the effect for $\widehat{N}_{e}$ is then the inverse of that for $1 / \widehat{N}_{e}$, e.g. underestimation instead of overestimation. Relative bias and coefficient of variation $(\mathrm{CV})$ of $1 / N_{\mathrm{e}}$ estimates were calculated as:

$$
\text { Relative bias }=\frac{\mu-1 / E\left[N_{e}\right]}{1 / E\left[N_{e}\right]} \quad C V=\frac{\sigma}{\mu},
$$

where $\mu$ was the mean and $\sigma$ the standard deviation of the $15001 / \widehat{N}_{e}$ estimates.

\subsection{Distribution of $N_{e}$ estimates}

For all simulated population sizes the interquartile range of relative estimates $\left(E\left[N_{e}\right] / \widehat{N}_{e}\right)$ decreased with sample size and for a given sample size was largest for the larger population sizes (Fig. 2, note 
different scales for y-axis). Estimates were generally positively biased though negative values occurred for the larger population sizes when sample size was small.

No negative $N_{e}$ estimates for population size $N=1000$ were found, whatever the sample size $S \geq 50$

(Fig. 3). For $N=10000$, only the smallest sample size $(S=50)$ led to negative $\widehat{N}_{e}$ estimates $(3.5 \%)$. For $N=100000$ and $N=1000000$, negative $\widehat{N}_{e}$ estimates were absent when respectively at least 1000 or 10 000 individuals were sampled, which represents $1 \%$ of $N$. For sample sizes $<100$ individuals (the most common sample size found in the literature review above), the percentage of negative $\widehat{N}_{e}$ reached a maximum of $53 \%$ for $S=50$ for $N=1000000$. Comparing results for $N=10000$ with $N=100000$, for sample size $S=50$, the number of negative $N_{e}$ estimates increased by $1618 \%$. The same comparison between $N=100000$ and $N=1000000$ showed an increase of $120 \%$ of negative estimates. Thus with usual samples sizes (Fig. 1), a population of 1000000 individuals could easily be evaluated having an infinite $N_{e}$ due to the high probability of obtaining a negative $N_{e}$ estimate $(>50 \%)$. Indeed, Zhivotovsky et al. (2016) attempted to estimate $N_{\mathrm{e}}$ for cod in the Barents Sea using a small sample ( $S=$ 43) and few microsatellites (13). As expected they found that all estimation methods gave negative $N_{e}$ estimates.

\subsection{Bias and precision of $N_{e}$ estimates}

In terms of relative bias, all simulated population sizes converged to a mean relative positive bias of around $+50 \%$ (Fig. 4a). For all $N$, precision increased (CV decreased) with increasing sample size (Fig. 4b). As expected, the worst precision was obtained for $N=1000000$ and $S=50$ for which $1 / \widehat{N}_{e}$ was overestimated as much as 88 times for certain replicates and samples. Globally for all simulated population sizes, given a sufficient sample size, the $\mathrm{CV}$ for $1 / \widehat{N}_{e}$ was smaller than 0.2 . Thus the sample sizes needed for stabilizing mean relative bias estimates and achieving a CV of less than 0.2 were around $1 \%$ of $N$ for $N \in(10000,100000$ and 1000000$)$. For $N=1000$ it was $S=50$ as we did not test smaller sample sizes. 


\subsection{Discussion}

Most marine fishes have overlapping generations and may have large population sizes (millions to billions of individuals), whereas genetic effective population size estimators generally assume discrete generations but also implicitly small population sizes. Using a simulation approach, we examined the feasibility of estimating the effective population size of a realistic fish species taking thornback ray as an example and using the popular LD method. For a given sample size, the results showed a large increase in the percentage of negative estimates with census population size. For example, in simulations for a population size of one million individuals, 200 SNPs and sample size 50 individuals, $53 \%$ of $N_{e}$ estimates were negative. This means that a study attempting to estimate $N_{\mathrm{e}}$ for a real thornback ray population of one million individuals would have a $50 \%$ chance of producing a negative estimate, which could lead to the wrong conclusion that the effective population size was very large, i.e. infinite. Thornback ray populations in the Northeast Atlantic are thought to range from half a million to more than three millions individuals (F. Marandel unpublished results). Thus the percentage of negative estimates of $N_{e}$ for a real thornback ray population can be expected to be even higher than what we found here if a sample of only 50 individuals is used. Waples (2016) simulated an ideal population of one million individuals and estimated $N_{e}$ with 5000 sampled individuals and 100 SNPs. In this case, the percentage of negative estimates reached also 50\%. Again this result for an ideal population corroborates that estimating $N_{e}$ for large real fish populations can be challenging already because of sampling difficulties. Moreover, in Waples (2016), even when $N_{e}$ was estimated to be positive, the values were underestimated by as much as $99 \%$. Thus, for real applications even when positive finite estimates of $N_{e}$ are found, these estimates can still be hugely biased and imprecise (Fig 4). Note that the simulations assumed perfect genotyping, any genotyping errors will further decrease precision.

The probability of obtaining negative $N_{e}$ estimates value can be reduced by increasing sample size. Our simulation study suggests that a sample size of around $1 \%$ of the census population size $N$ might be sufficient to obtain precise (but biased) estimates using LDM, which at the same time avoids negative estimates. However, in the case of ray populations this means that appropriate samples sizes 
can reach several thousands of individuals. Much larger sample sizes might be necessary for teleost fish populations which obviously limits the economic and logistic feasibility of genetic effective population size studies.

A single sample method such as the LD method can easily be applied opportunistically in studies where $N_{e}$ estimation is a side goal (for example, in population genetics studies), and thus, rely on small sample sizes that are not fit for this purpose. For example, Watson et al. (2016) studied the population genetic structure of the European lobster in the Irish Sea jointly with the estimation of $N_{e}$ for nine sampling locations. For six locations using the LD method, $N_{e}$ was estimated to be negative (with confidence intervals including infinity) and thus interpreted to be infinite. The sample sizes used in this study varied between 29 and 48 individuals which suggests that the negative $N_{e}$ estimates were a consequence of the small sample sizes used rather than infinite effective population size.

In this study simulations were carried out for a thornback ray like species. While $1 / \widehat{N}_{e}$ estimates were rather variable we found that for an appropriate sample size, the mean relative bias was around $+50 \%$. As overestimation of $1 / \widehat{N}_{e}$ means that $\widehat{N}_{e}$ is underestimated, a $50 \%$ overestimation of $1 / \widehat{N}_{e}$ corresponds to an underestimation of $\widehat{N}_{e}$ of around $31 \%$. The existence of underestimation is a wellknown property of the LD method for species with overlapping generations (Waples et al. 2014). The reported amount of underestimation for random samples of adults lies between 50\% (mosquito) and $10 \%$ (cod) (Waples et al. 2014) with the $30 \%$ found for a ray like species for random samples of newborns lying in between. Assuming the simulations were sufficiently realistic for thornback ray, the correction of $N_{e}$ estimates obtained with the LDM for a thornback ray like population might be attempted, but only if a sufficiently large sample size was used.

We now briefly discuss the assumptions made in the simulation study and their possible impacts on the results. Populations were simulated for 151 years and newborns were sampled in the final year only to estimate $N_{e}$ with the LDM. The 150 first years can be considered a long burn-in to ensure reaching the equilibrium for population dynamics but also for the allele frequencies of the genetic markers. We used 200 SNPs with an allele frequency of 0.5 at the start. Using more SNPs might increase precision (Waples and Do 2010), though initial trials showed that the gain should be small, while using a different allele frequency, i.e. $<0.5$ minor allele frequency, would lead to more SNPs 
being excluded due to thresholding (SNPs with minor allele frequency $<0.05$ in the last year were excluded). No physical link between SNPs was assumed; technically this was achieved by coding each SNP on a different chromosome. This is an ideal situation which is not likely to happen when using empirical genetic markers. Physical linkage is expected to increase the downward bias of $\widehat{N_{e}}$ estimates (Waples et al. 2016). Further, we only used samples from newborns but results were similar using samples stratified by age for all ages or only mature ones (F. Marandel results not shown). We only studied the effect of sample size and its interaction with census population size and ignored other sources of errors such as genotyping errors, particular genomic or ecological features such as polyploidization, which will also impact real life estimates and probably imply that even larger samples are needed to stabilize bias and precision. Lastly, only the LDM was used for estimating $N_{e}$. Numerous other genetic estimators are available (see Wang 2016 for a complete review) but all are expected to perform poorly for small sample sizes (and several need corrections for overlapping generations).

\section{CONCLUDING REMARKS}

Numerous methods for estimating effective population size are available but they all suffer from different sources of bias and uncertainty. They also all demand high sampling effort, sometimes explicitly (e.g. the temporal method requires several samples separated in times) and sometimes implicitly (e.g. the Linkage Disequilibrium method requires a large number of individuals to be sampled). The amount of bias in genetic estimates of effective population size depends on the life history traits of the studied species (Waples et al. 2014). Thus, particular attention should be paid to the interpretation of positive finite $\widehat{N_{e}}$ estimates as large underestimation or overestimation can occur. Moreover, due to large population sizes in the marine environment, negative $N_{e}$ estimates are commonly found and should be interpreted with care as they might indicate insufficient sample sizes rather than infinite true $N_{e}$. In our simulations, for $N=1000000$ and $S=50$, half of all replicates led to negative $N_{e}$ estimates suggesting sample size was insufficient. However, if by chance a positive finite 
$N_{e}$ is estimated, it cannot be interpreted as a proof that the sample size is sufficient as half of the estimates were indeed positive for this sample size.

While theoretically it might be possible to correct $N_{\mathrm{e}}$ estimates, in practice at least two conditions need to be met. First, simulations reproducing the species life history sufficiently well will need to be carried out to estimate a species-specific bias correction factor. For a thornback ray like species we found NeEstimator underestimated $N_{\mathrm{e}}$ by $31 \%$, while Waples et al. (2014) found a $10 \%$ bias for cod. Second, a sufficiently large number of individuals needs to be sampled, probably around $1 \%$ or more of census population size. While the first condition is time consuming, it remains feasible. However, given the large population sizes of many marine fishes, sampling $1 \%$ will require samples sizes which are often neither practical nor financially feasible. Thus, while the effective population size concept is suitable for evaluating the genetic status of marine populations, popular tools and sampling designs often miss the target (small sample size, too large population). If however precise bias-corrected estimates of effective population size can be obtained, declines in $N_{e}$ track declines in $N$ and thus, can be informative for management (Ovenden et al. 2016).

In conclusion, for large marine populations either appropriate sample sizes are used or $N_{e}$ should not be estimated and reported. This study found that for a thornback ray like species sample size should be around $1 \%$ of absolute population size for the Linkage Disequilibrium method. If however such large samples are collected, other population quantities can be estimated using the same data. Absolute abundance and demographic parameters (fecundity, mortality) can be estimated with the close-kin mark-recapture (CKMR) method (Bravington et al. 2016a, 2016b). This method is based on the identification of pairs of close relatives (parents-offspring or half sibling pairs). Pairs of related individuals sampled at different locations can also on inform on migration (Feutry et al. 2017) and be used for estimating $N_{e}$ (Waples et al. 2018). However, as these approaches have not been much used, further studies are needed to evaluate their merits and limits. 


\section{Acknowledgements}

We acknowledge funding from the French "Agence Nationale de la Recherche" (ANR) for the GenoPopTaille project and the Fondation Total (project GenoPopTaille-Capsules). FM thanks Ifremer for a PhD studentship and a grant who allowed her to work at the NOAA of Seattle. FM tanks Bo Peng for his help with simuPOP code. We would like to thank two anonymous reviewers for constructive comments which helped to improve the manuscript.

\section{References}

Bravington, M.V., Grewe, P.M., and Davies, C.R. 2016a. Absolute abundance of southern bluefin tuna estimated by close-kin mark-recapture. Nat. Commun. 7: 13162. doi:10.1038/ncomms 13162.

Bravington, M.V., Skaug, H.J., and Anderson, E.C. 2016b. Close-Kin Mark-Recapture. Stat. Sci. 31(2): 259-274. doi:10.1214/16-STS552.

Bryan-Brown, D., Brown, C., Hughes, J., and Connolly, R. 2017. Patterns and trends in marine population connectivity research. Mar. Ecol. Prog. Ser. 585: 243-256. doi:10.3354/meps12418.

Chevolot, M., Ellis, J.R., Rijnsdorp, A.D., Stam, W.T., and Olsen, J.L. 2008. Temporal changes in allele frequencies but stable genetic diversity over the past 40 years in the Irish Sea population of thornback ray, Raja clavata . Heredity 101(2): 120-126. doi:10.1038/hdy.2008.36.

Dankel, D.J., and Edwards, C.T.T. 2016. Fishery systems and the role of management science. In Management science in fisheries: an introduction to simulation-based methods. Routledge, London: New York. pp. 3-15.

DeWoody, J.A., Fernandez, N.B., Brüniche-Olsen, A., Antonides, J.D., Doyle, J.M., San Miguel, P., Westerman, R., Vertyankin, V.V., Godard-Codding, C.A.J., and Bickham, J.W. 2017. Characterization of the Gray Whale Eschrichtius robustus Genome and a Genotyping Array Based on Single-Nucleotide Polymorphisms in Candidate Genes. Biol. Bull. 232(3): 186-197. doi:10.1086/693483.

Do, C., Waples, R.S., Peel, D., Macbeth, G.M., Tillett, B.J., and Ovenden, J.R. 2014. NeEstimator V2: re-implementation of software for the estimation of contemporary effective population size $\mathrm{Ne}$ from genetic data. Mol. Ecol. Resour. 14(1): 209-214. doi:10.1111/1755-0998.12157.

Dudgeon, C.L., Blower, D.C., Broderick, D., Giles, J.L., Holmes, B.J., Kashiwagi, T., Krück, N.C., Morgan, J.A.T., Tillett, B.J., and Ovenden, J.R. 2012. A review of the application of molecular genetics for fisheries management and conservation of sharks and rays. J. Fish Biol. 80(5): 1789-1843. doi:10.1111/j.1095-8649.2012.03265.x.

Dudgeon, C.L., and Ovenden, J.R. 2015. The relationship between abundance and genetic effective population size in elasmobranchs: an example from the globally threatened zebra shark Stegostoma fasciatum within its protected range. Conserv. Genet. 16(6): 1443-1454. doi:10.1007/s10592-015-0752-y.

Feutry, P., Berry, O., Kyne, P.M., Pillans, R.D., Hillary, R.M., Grewe, P.M., Marthick, J.R., Johnson, G., Gunasekera, R.M., Bax, N.J., and Bravington, M. 2017. Inferring contemporary and historical genetic connectivity from juveniles. Mol. Ecol. 26(2): 444-456. doi: $10.1111 / \mathrm{mec} .13929$.

Hamilton, D.C., and Cole, D.E.C. 2004. Standardizing a Composite Measure of Linkage Disequilibrium. Ann. Hum. Genet. 68(3): 234-239. doi:10.1046/j.1529-8817.2004.00056.x.

Hare, M.P., Nunney, L., Schwartz, M.K., Ruzzante, D.E., Burford, M., Waples, R.S., Ruegg, K., and Palstra, F. 2011. Understanding and estimating effective population size for practical 
application in marine species management: applying effective population size estimates to marine species management. Conserv. Biol. 25(3): 438-449. doi:10.1111/j.15231739.2010.01637.x.

Hedgecock, D. 1994. Does variance in reproductive success limit effective population size of marine organisms? In Genetics and evolution of aquatic organisms, Chapman and Hall. Beaumont M, London. pp. 122-134.

Hill, W.G. 1981. Estimation of effective population size from data on linkage disequilibrium. Genet. Res. 38(03): 209-216.

Hoarau, G., Boon, E., Jongma, D.N., Ferber, S., Palsson, J., Van der Veer, H.W., Rijnsdorp, A.D., Stam, W.T., and Olsen, J.L. 2005. Low effective population size and evidence for inbreeding in an overexploited flatfish, plaice (Pleuronectes platessa L.). Proc. R. Soc. B Biol. Sci. 272(1562): 497-503. doi:10.1098/rspb.2004.2963.

Holland, L.P., Jenkins, T.L., and Stevens, J.R. 2017. Contrasting patterns of population structure and gene flow facilitate exploration of connectivity in two widely distributed temperate octocorals. Heredity 119(1): 35-48. doi:10.1038/hdy.2017.14.

Jorde, P.E., and Ryman, N. 1995. Temporal allele frequency change and estimation of effective size in populations with overlapping generations. Genetics 139(2): 1071090.

Laconcha, U., Iriondo, M., Arrizabalaga, H., Manzano, C., Markaide, P., Montes, I., Zarraonaindia, I., Velado, I., Bilbao, E., Goñi, N., Santiago, J., Domingo, A., Karakulak, S., Oray, I., and Estonba, A. 2015. New Nuclear SNP Markers Unravel the Genetic Structure and Effective Population Size of Albacore Tuna (Thunnus alalunga). PLOS ONE 10(6): e0128247. doi:10.1371/journal.pone.0128247.

Laurent, V., and Planes, S. 2007. Effective population size estimation on Sardina pilchardus in the Bay of Biscay using a temporal genetic approach: effective population sizes of sardines. Biol. J. Linn. Soc. 90(4): 591-602. doi:10.1111/j.1095-8312.2007.00747.x.

Laurie-Ahlberg, C.C., and Weir, B.S. 1979. Allozymic Variation and Linkage Disequilibrium in Some Laboratory Populations of Drosophila melanogaster. Genetics 92(4): 1295-1314.

Leberg, P. 2005. Genetic approaches for estimating the effective size of populations. J. Wildl. Manag. 69(4): 1385-1399. doi:10.2193/0022-541X(2005)69[1385:GAFETE]2.0.CO;2.

Luikart, G., Ryman, N., Tallmon, D.A., Schwartz, M.K., and Allendorf, F.W. 2010. Estimation of census and effective population sizes: the increasing usefulness of DNA-based approaches. Conserv. Genet. 11(2): 355-373. doi:10.1007/s10592-010-0050-7.

Macbeth, G.M., Broderick, D., Buckworth, R.C., and Ovenden, J.R. 2013. Linkage Disequilibrium Estimation of Effective Population Size with Immigrants from Divergent Populations: A Case Study on Spanish Mackerel (Scomberomorus commerson). G3amp58 GenesGenomesGenetics 3(4): 709-717. doi:10.1534/g3.112.005124.

Marandel, F., Lorance, P., and Trenkel, V.M. 2016. A Bayesian state-space model to estimate population biomass with catch and limited survey data: application to the thornback ray (Raja clavata) in the Bay of Biscay. Aquat. Living Resour. 29(2): 209. doi:10.1051/alr/2016020.

Montes, I., Iriondo, M., Manzano, C., Santos, M., Conklin, D., Carvalho, G.R., Irigoien, X., and Estonba, A. 2016. No loss of genetic diversity in the exploited and recently collapsed population of Bay of Biscay anchovy (Engraulis encrasicolus, L.). Mar. Biol. 163(5). doi:10.1007/s00227-016-2866-2.

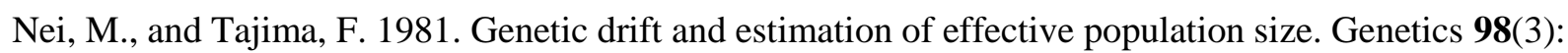
625-640.

Ovenden, J.R., Berry, O., Welch, D.J., Buckworth, R.C., and Dichmont, C.M. 2015. Ocean's eleven: a critical evaluation of the role of population, evolutionary and molecular genetics in the management of wild fisheries. Fish Fish. 16(1): 125-159. doi:10.1111/faf.12052.

Ovenden, J.R., Leigh, G.M., Blower, D.C., Jones, A.T., Moore, A., Bustamante, C., Buckworth, R.C., Bennett, M.B., and Dudgeon, C.L. 2016. Can estimates of genetic effective population size contribute to fisheries stock assessments? J. Fish Biol. 89(6): 2505-2518. doi:10.1111/jfb.13129.

Peng, B., and Kimmel, M. 2005. simuPOP: a forward-time population genetics simulation environment. Bioinformatics 21(18): 3686-3687. doi:10.1093/bioinformatics/bti584. 
Pita, A., Pérez, M., Velasco, F., and Presa, P. 2017. Trends of the genetic effective population size in the Southern stock of the European hake. Fish. Res. 191: 108-119. doi:10.1016/j.fishres.2017.02.022.

Poulsen, N.A., Nielsen, E.E., Schierup, M.H., Loeschcke, V., and GrøNkjaer, P. 2005. Long-term stability and effective population size in North Sea and Baltic Sea cod (Gadus morhua): effective population size in atlantic cod. Mol. Ecol. 15(2): 321-331. doi:10.1111/j.1365294X.2005.02777.x.

Schwartz, M.K., Tallmon, D.A., and Luikart, G. 1998. Review of DNA-based census and effective population size estimators. Anim. Conserv. 1(4): 293-299. doi:10.1111/j.14691795.1998.tb00040.x.

Soulé, M. 1987. Viable Populations for Conservation. Cambridge University Press, Cambridge, UK.

Wang, J. 2001. A pseudo-likelihood method for estimating effective population size from temporally spaced samples. Genet. Res. 78(3): 243-257.

Wang, J. 2005. Estimation of effective population sizes from data on genetic markers. Philos. Trans. R. Soc. B Biol. Sci. 360(1459): 1395-1409. doi:10.1098/rstb.2005.1682.

Wang, J. 2009. A new method for estimating effective population sizes from a single sample of multilocus genotypes. Mol. Ecol. 18(10): 2148-2164. doi:10.1111/j.1365-294X.2009.04175.x.

Wang, J. 2016. A comparison of single-sample estimators of effective population sizes from genetic marker data. Mol. Ecol. 25(19): 4692-4711. doi:10.1111/mec.13725.

Waples, R.K., Larson, W.A., and Waples, R.S. 2016. Estimating contemporary effective population size in non-model species using linkage disequilibrium across thousands of loci. Heredity 117(4): 233-240. doi:10.1038/hdy.2016.60.

Waples, R.S. 2006. A bias correction for estimates of effective population size based on linkage disequilibrium at unlinked gene loci*. Conserv. Genet. 7(2): 167-184. doi:10.1007/s10592005-9100-y.

Waples, R.S. 2016. Tiny estimates of the $N_{\mathrm{e}} / N$ ratio in marine fishes: Are they real? J. Fish Biol. 89(6): 2479-2504. doi:10.1111/jfb.13143.

Waples, R.S., Antao, T., and Luikart, G. 2014. Effects of overlapping generations on Linkage Disequilibrium estimates of effective population size. Genetics 197(2): 769-780. doi:10.1534/genetics.114.164822.

Waples, R.S., and Do, C. 2010. Linkage disequilibrium estimates of contemporary Ne using highly variable genetic markers: a largely untapped resource for applied conservation and evolution. Evol. Appl. 3(3): 244-262. doi:10.1111/j.1752-4571.2009.00104.x.

Waples, R.S., Do, C., and Chopelet, J. 2011. Calculating $N_{\mathrm{e}}$ and $N_{\mathrm{e}} / N$ in age-structured populations: a hybrid Felsenstein-Hill approach. Ecology 92(7): 1513-1522. doi:10.1890/10-1796.1.

Waples, R.S., Grewe, P.M., Bravington, M.W., Hillary, R., and Feutry, P. 2018. Robust estimates of a high $N_{\mathrm{e}} / N$ ratio in a top marine predator, southern bluefin tuna. Sci. Adv. 4(7): eaar7759. doi:10.1126/sciadv.aar7759.

Watson, H.V., McKeown, N.J., Coscia, I., Wootton, E., and Ironside, J.E. 2016. Population genetic structure of the European lobster (Homarus gammarus) in the Irish Sea and implications for the effectiveness of the first British marine protected area. Fish. Res. 183: 287-293. doi:10.1016/j.fishres.2016.06.015.

Weir, B.S. 1979. Inferences about Linkage-Disequilibrium. Biometrics 35: 235-254.

Zhivotovsky, L.A., Teterina, A.A., Mukhina, N.V., Stroganov, A.N., Rubtsova, G.A., and Afanasiev, K.I. 2016. Effects of genetic drift in a small population of Atlantic cod (Gadus morhua kildinensis Derjugin) landlocked in a meromictic lake: genetic variation and conservation measures. Conserv. Genet. 17(1): 229-238. doi:10.1007/s10592-015-0774-5. 


\section{Competing interests}

We have no competing interests.

\section{Funding}

The study received funding from the French "Agence Nationale de la Recherche" (project GenoPopTaille, contract ANR-14-CE02-0006-01), the Fondation Total (project GenoPopTailleCapsules) and the European Union (projet Pandora).

\section{Tables}

Table 1: Simulation design. $N$ is the simulated population size used in simuPOP; $E\left[N_{e}\right]$ is the expected $N_{e}$ estimated with AgeNe.

\begin{tabular}{l|l|l}
\hline Simulated $\boldsymbol{N}$ & $\mathbf{E}\left[\boldsymbol{N}_{e}\right]$ & Tested sample sizes $(\boldsymbol{S})$ \\
\hline 1000 & 87 & $50,100,200,300$ \\
10000 & 870 & $50,100,200,300,500,1000$ \\
100000 & 8700 & $50,100,200,300,500,1000,1500$ \\
1000000 & 87000 & $50,100,200,300,500,1000,1500,5000,10000$ \\
\hline
\end{tabular}




\section{Figures}

Figure 1: Meta-analysis of literature reported estimates of effective population size $\left(\widehat{N}_{e}\right)$ in relation to sample size for a) studies with $N_{e}$ estimation as main goal and c) studies with other goals, and in relation to census population size for b) studies with $N_{e}$ estimation as main goal and d) studies with other goals. Infinite $N_{e}$ estimates $(\infty)$ in the original publications were plotted at 30000 while reported negative estimates were plotted in grey at 30000 . Sources for census population size estimates are provided in table S2. Points in common between panels a), b) and panels c),d) are filled in.
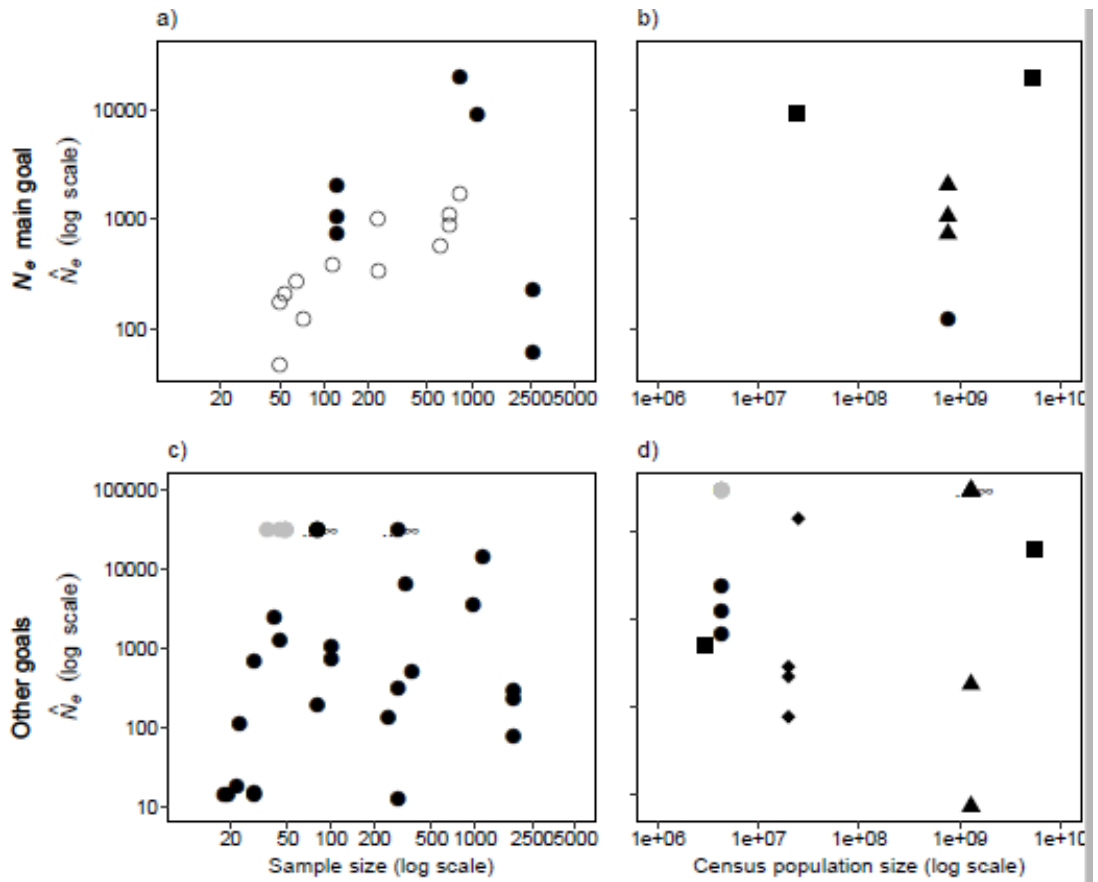

Census population size estimate available $\bigcirc$ No - Yes

$N_{e}$ estimates - Positive estimates - Negative estimates 
Figure 2: Ratio of inverse effective population size $1 / \widehat{N_{e}}$ estimated with the Linkage Disequilibrium method for simulated genetic samples using NeEstimator (Do et al. 2014) and expected effective population size $1 / E\left[N_{e}\right]$ for chosen simulation parameters calculated by AgeNe (Waples et al. 2011). Sample sizes go from 50 to 10000 individuals (newborns). Panels: simulated population size. Box: 75 and 25 percentiles, vertical line: 95 and 5 percentiles; horizontal bar: mean estimates. Dashed line: $1 / \widehat{N_{e}}=1 / E\left[N_{e}\right]$

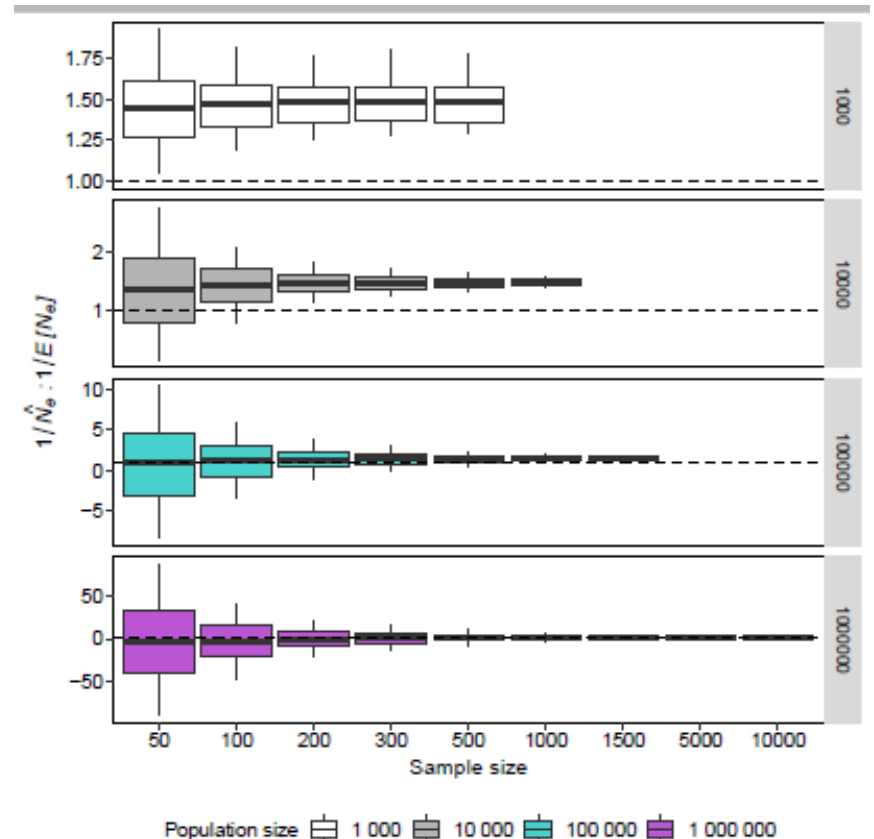

Figure 3: Percentage of negative effective population size estimates $\left(\widehat{N}_{e}\right)$ for a simulated thornback ray like population estimated with the Linkage Disequilibrium method (NeEstimator, Do et al. 2014). Shapes: simulated population size.

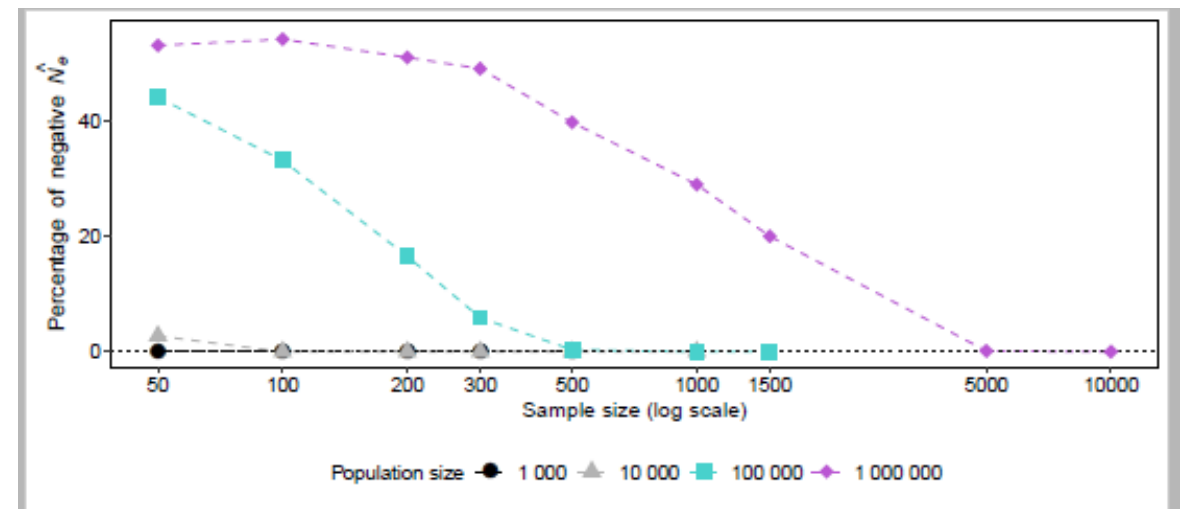


Figure 4: a) Relative bias and b) CV of inverse effective population size estimates $\left(1 / \widehat{N_{e}}\right)$ calculated by Linkage Disequilibrium method for simulated genetic samples using NeEstimator (Do et al. 2014). Relative bias is with respect to expected effective population size $1 / E\left[N_{e}\right]$ for chosen simulation parameters calculated by AgeNe (Waples et al. 2011). Sample sizes go from 50 to 10000 individuals (newborns). Shapes: simulated population size.
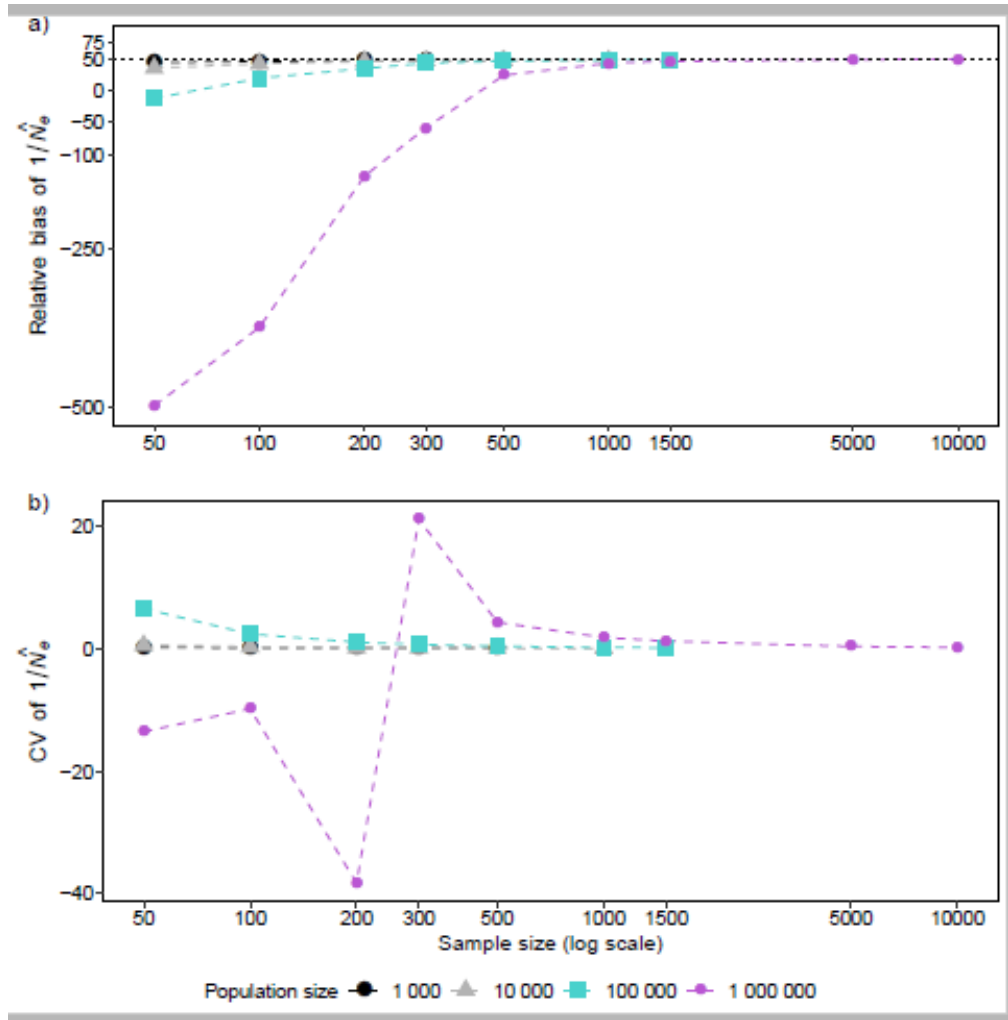